\title{
The Computer System for Monitoring of Induction Motors Condition of Mining Machinery
}

\author{
Veniamin Kashirskikh ${ }^{1,}$, Valery Zavyalov ${ }^{2}$, Irina Semykina $^{1}$, and Valery Borovtsov ${ }^{1}$ \\ ${ }^{1}$ T.F. Gorbachev Kuzbass State Technical University, Department of Electric Drive and Automation, \\ 650000, Kemerovo, Russia \\ ${ }^{2}$ National Research Tomsk Polytechnic University, Department of Electric Drive and Automation, \\ 634050, Tomsk, Russia
}

\begin{abstract}
The possibility of carrying out monitoring of parameters and variables of the induction electric motor (IM) working as a part of the electric mining machinery drive is considered. Monitoring is carried out on the working inventory in real time by means of the computer system on the basis of the IM mathematical model, mathematical estimation methods and also information which is contained in easily measured phase tension and IM stator currents. As a result IM indicators are defined difficult or cannot be measured in the mining machinery operation course. These are the active and induced windings resistances of a stator and a rotor, a magnetic linkage, an angular frequency of a rotor rotation and the electromagnetic moment. Algorithms of dynamic IM identification and the software are developed for carrying out monitoring. Theoretical provisions and conclusions are confirmed with results of the pilot studies. Information received during monitoring can be used for perfecting of the electric drive.
\end{abstract}

\section{Introduction}

Monitoring of induction motors condition in the course of their work on the basis of dynamic identification can be used for further perfecting of asynchronous electric drives of mining machinery. The information obtained during monitoring is necessary for better management, functional diagnosing and IM protection and asynchronous electric drive for the emergency cycles.

The IM condition is understood some set of its parameters, output and intermediate variables and their derivatives which change in time in function for entrance influences. It is apparent that control system overall performance of the adjustable electric drive depends on knowledge of the current values of rotor rotation angular frequency, the electromagnetic moment and electromagnetic parameters of the motor - the active and induced resistance of IM windings. Parameters values given in catalogs are calculations at projection and their use for management leads to mistakes.

Besides, actual values of parameters depend on operating regime and a thermal condition of IM. It is known that in the mode of direct start-up the active rotor resistance can change more than by 1.5 times, and inductances - for $30-40 \%$. The active resistance of

${ }^{*}$ Corresponding author : kvg.ea@kuzstu.ru 
a stator winding can change during the IM work for $20-30 \%$ that is especially characteristic for the repeated and short-term mode. Therefore, the current values of these parameters need to be defined in the course of IM work. However measurement of the active resistance and rotor inductance, chain inductance of magnetization and magnetic linkage of stator and rotor is in essence impossible, and there are also inaccessible stator parameters for direct measurement in the course of IM work. The rotor rotation frequency can be measured at motor run, however during the operation at the mining enterprises it is accompanied by the considerable technical difficulties. All this means the need of indirect methods use of a IM condition monitoring, for example, the dynamic identification aims in obtaining necessary information by means of the computer information processing which is contained in the measured tension and stator currents on the basis of the IM mathematical model and mathematical estimation methods.

\section{Materials and methods}

The great amount of publications on identification of certain parameters and variables of electric motors condition for various modes and also technical solutions for their realization is known [1-6]. However the creation aim of the computer system allowing to calculate in real time the current values of parameters and variables of a IM condition inaccessible for measurement demands the same way to development of special algorithms for dynamic IM identification having low sensitivity to a hum level and their statistical characteristics, and providing computing processes stability of estimation.. The theoretical basis for the solution of these tasks is given in [7-13].

Various estimation methods described in $[14,15]$ were used for carrying out dynamic identification taking into account the given requirements. The use for these purposes of the recursive least-square method (RLSM) and the extended Kalman filter (EKF) is considered here. The mathematical model widely used for the solution of various theoretical and practical tasks on the equations basis of the two-phase generalized electrical machine (GEM) [16,17] was taken as the mathematical IM model which can be presented in a complex form by set of the following equations:

$$
\begin{array}{cc}
U_{1}=I_{1} R_{1}+\dot{\Psi}_{1}+j \omega_{k} \Psi_{1} ; & 0=I_{2} R_{2}+\dot{\Psi}_{2}+j\left(\omega_{k}-p \omega_{r}\right) \Psi_{2} ; \\
M=p \operatorname{Im}\left(\Psi_{1}^{*} I_{1}\right) ; & \dot{\omega}_{r}=\frac{1}{J}\left(M-M_{c}\right) ; \\
\Psi_{1}=I_{1} L_{1}+I_{2} L_{m} ; & \Psi_{2}=I_{2} L_{2}+I_{1} L_{m} ; \\
L_{1}=L_{m}+L_{1 \sigma} ; & L_{2}=L_{m}+L_{2 \sigma},
\end{array}
$$

where $U_{l}, U_{2}, I_{1}, I_{2}, \Psi_{1}, \Psi_{2}$ - respectively vectors of tension, currents and magnetic linkages of windings of a stator and rotor; $\omega_{k}, \omega_{r}$ - angular rotation frequencies of coordinate axes and a rotor; $R_{1}, R_{2}, L_{1}, L_{2}$ - the active resistance and windings inductances of a stator and a rotor; $L_{m}$ - inductance of a magnetization chain; $L_{1 \sigma}, L_{2 \sigma}$ - leakage inductances of windings of a stator and a rotor; $M$ - the electromagnetic moment; $M_{c}-$ section modulus on a shaft; $J$ - rotor moment of inertia; $p$ - poles couples number.

Hereinafter the index 1 corresponds to belonging of parameter or a variable to a stator and the index 2 - to a rotor. The index $(*)$ shows that the vector is the conjugate, and the point over a variable designates its derivative. The rotor winding is brought to a stator winding. The accepted assumptions correspond to reference assumptions of GEM. In many publications it is noted that the accuracy of decisions received when using by GEM is quite sufficient for the majority of practical tasks. 
The complex of algorithms developed on the basis of RLSM and EKF with the use of the mathematical models of a IM condition and a chain of measurement includes estimation algorithms of separate vector parameters components and a condition of electric motors and the software. In the beginning the preliminary IM identification is carried out and as a result of which the magnetization curve parameters of a magnetic circuit, the active resistance value and a magnetic linkage of a stator are defined on the basis of the methods developed by us. At this stage also the rotor inertia moment is defined by the known way.

Different algorithms of identification are developed for static and dynamic duties of a IM because the measured values of tension and currents will contain at the same time different amount of information about parameters and variables of a state. The mode dynamism degree definition is made by means of the numerical criterion considering the power of the first harmonica of a magnetic stator linkage. The researches showed that at $K_{p 1}>0.97$ the mode can be accepted static with use of the corresponding algorithms.

For carrying out identification the mathematical models of a state and measurement chain of a IM were received taking into account regularities of the physical processes happening in a IM and use of information which is contained in the measured rotor rotation frequencies, phase tension and currents of a stator. Definition of vectors covariance matrixes elements of a state error and measurement error during estimation is based on the experimental data obtained by measurements of noise in a measuring system at the switched-off motor.

For example, for a static IM duty the value determination of the active rotor resistance is based on use the following equations as mathematical model of an IM condition:

$$
\left.\begin{array}{l}
\dot{\Psi}_{2 \alpha}=-\frac{R_{2}}{L_{2}} \Psi_{2 \alpha}-p \omega_{r} \Psi_{2 \beta}+\frac{R_{2} L_{m}}{L_{2}} i_{1 \alpha} ; \\
\dot{\Psi}_{2 \beta}=-\frac{R_{2}}{L_{2}} \Psi_{2 \beta}+p \omega_{r} \Psi_{2 \alpha}+\frac{R_{2} L_{m}}{L_{2}} i_{1 \beta}
\end{array}\right\}
$$

with their subsequent reduction to a discrete type and expansion of state vector by including active rotor resistance in it.

Two variants were considered to carry out this procedure. The analytical solution of equations represented in the matrix form with reduction to mathematical state model is used in the first variant:

$$
x_{e}=\left[\begin{array}{lll}
\Psi_{2 \alpha} \Psi_{2 \beta} & R_{2}
\end{array}\right]^{T} ; \quad f\left(x_{e}, z\right)=\left[\begin{array}{l}
f_{1} \\
f_{2} \\
f_{3}
\end{array}\right]=\left[\begin{array}{l}
a_{1} \Psi_{2 \alpha}-a_{2} \Psi_{2 \beta}+a_{3} i_{2 \alpha}-a_{4} i_{2 \beta} \\
a_{2} \Psi_{2 \alpha}+a_{1} \Psi_{2 \beta}+a_{4} i_{2 \alpha}+a_{3} i_{2 \beta} \\
R_{2}
\end{array}\right]
$$

where $a_{1}, a_{2}, a_{3}, a_{4}-$ the coefficients depending on rotor parameters, inductance of a magnetization chain and a rotor rotation frequency; $\Psi_{2 \alpha}, \Psi_{2 \beta}$ - the making rotor magnetic linkages.

In the second variant the approximation of the equations given above was used to a rotor chain on the basis of a numerical method of Euler with their transformation to a discrete state and as a result receiving of mathematical model of an IM condition in the form of: 


$$
x_{e}=\left[\begin{array}{lll}
\Psi_{2 \alpha} & \Psi_{2 \beta} & R_{2}
\end{array}\right]^{T} ; \quad f\left(x_{e}, z\right)=\left[\begin{array}{l}
f_{1} \\
f_{2} \\
f_{3}
\end{array}\right]=\left[\begin{array}{l}
\Psi_{2 \alpha}+\left(-\frac{R_{2}}{L_{2}} \Psi_{2 \alpha}-\omega_{r} \Psi_{2 \beta}+\frac{R_{2} L_{m}}{L_{2}} i_{l \alpha}\right) T_{k} \\
\Psi_{2 \beta}+\left(-\frac{R_{2}}{L_{2}} \Psi_{2 \beta}+\omega_{r} \Psi_{2 \alpha}+\frac{R_{2} L_{m}}{L_{2}} i_{l \beta}\right) T_{k} \\
R_{2}
\end{array}\right] .
$$

The mathematical model of a measurement chain for determination of the active rotor resistance has the following form:

$$
y=\left[\Psi_{2 \alpha} \Psi_{2 \beta}\right]^{T} ; \quad h\left(x_{e}, z\right)=\left[\begin{array}{l}
h_{1} \\
h_{2}
\end{array}\right]=\left[\begin{array}{l}
\frac{L_{2}}{L_{m}} \Psi_{1 \alpha}-\frac{L_{1} L_{2}-L_{m}^{2}}{L_{m}} i_{l \alpha} \\
\frac{L_{2}}{L_{m}} \Psi_{1 \beta}-\frac{L_{1} L_{2}-L_{m}^{2}}{L_{m}} i_{1 \beta}
\end{array}\right]
$$

The set of mathematical models of a state and IM measurement chain for parameters vector identification and a state in a static duty is received as a result of application of RLSM and EKF: $\theta_{s}=\left[\begin{array}{lllllll}\Psi_{1} & \Psi_{m} & \Psi_{2} & R_{2} & L_{1 \sigma} & L_{2} & L_{m}\end{array}\right]^{T}$

Unlike the static mode during the IM work in the dynamic mode it is possible simultaneous determination of the active rotor resistance and its rotation frequency. Identification of parameters and IM condition variables in this case is based on use of EKF and information which is contained only in the phase tension and currents of a stator. After initial transformation equations to difference equations and expansions of state vector with addition in it of moment resistance and the active resistance of a stator and a rotor, the mathematical model of an IM condition will take a form:

$$
f\left(x_{e}, z\right)=\left[\begin{array}{c}
x_{e}=\left[\Psi_{2 \alpha} \Psi_{2 \beta} \omega_{r} R_{1} R_{2} M_{c}\right]^{T} ; \\
f_{2} \\
f_{3} \\
f_{4} \\
f_{5} \\
f_{6}
\end{array}\right]=\left[\begin{array}{l}
\Psi_{2 \alpha}+\left(-\frac{R_{2}}{L_{2}} \Psi_{2 \alpha}-p \omega_{r} \Psi_{2 \beta}+\frac{R_{2} L_{m}}{L_{2}} i_{l \alpha}\right) T_{k} \\
\Psi_{2 \beta}+\left(-\frac{R_{2}}{L_{2}} \Psi_{2 \beta}+p \omega_{r} \Psi_{2 \alpha}+\frac{R_{2} L_{m}}{L_{2}} i_{l \beta}\right) T_{k} \\
\omega_{r}+\frac{1}{J}\left(\frac{3}{2} \frac{L_{m}}{L_{2}} p\left(\Psi_{2 \alpha} i_{l \beta}-\Psi_{2 \beta} i_{l \alpha}\right)-M_{c}\right) T_{k} \\
R_{1} \\
R_{2} \\
M_{c}
\end{array}\right] .
$$

The mathematical model of a measurement chain for this case was received at the combined solution of the equation used for definition of a magnetic linkage of a rotor and initial equations for receiving model of an IM condition at estimation of the active rotor resistance on the basis of EKF: 


$$
y=\left[\begin{array}{ll}
u_{1 \alpha} u_{1 \beta}
\end{array}\right]^{T} ; \quad h\left(x_{e}, u\right)=\left[\begin{array}{l}
\dot{i}_{1} \\
h_{2}
\end{array}\right]=\left[\begin{array}{l}
-\frac{R_{2} L_{m}}{L_{2}^{2}} \Psi_{2 \alpha}-p \omega_{r} \frac{L_{m}}{L_{2}} \Psi_{2 \beta}+ \\
+\left(R_{1}+R_{2} \frac{L_{m}^{2}}{L_{2}^{2}}\right) i_{l \alpha}+\frac{L_{1} L_{2}-L_{m}^{2}}{L_{2}} \dot{i}_{l \alpha} \\
-\frac{R_{2} L_{m}}{L_{2}^{2}} \Psi_{2 \beta}-p \omega_{r} \frac{L_{m}}{L_{2}} \Psi_{2 \alpha}+ \\
+\left(R_{1}+R_{2} \frac{L_{m}^{2}}{L_{2}^{2}}\right) i_{1 \beta}+\frac{L_{1} L_{2}-L_{m}^{2}}{L_{2}} \dot{i}_{l \beta}
\end{array}\right] .
$$

The way considered allows to define parameters vector and an IM condition in the

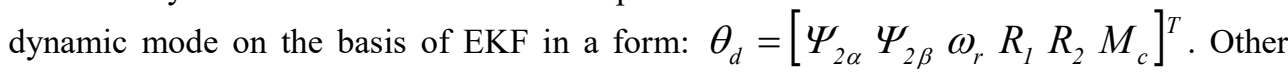
components of a vector are defined at a stage of preliminary identification on the basis of the data obtained at IM start-up idle in the mode of idle operation and during the IM work in the static mode. These procedures have to be done for each motor in advance, in the course of monitoring system preparation for operation.

The coordinate converter of tension and stator currents from a three-phase in two-phase system, the calculator of a derivative for the making stator currents and parameters calculators and IM variables realized in the program way are provided for algorithms work of estimation as a part of the computer system.

\section{Experiments}

The pilot algorithms studies of dynamic IM identification were carried out for an IM number with a power from 1 to $3 \mathrm{~kW}$ at the computerized stand which is special developed for these purposes., Parameter estimation results of the electric motor 4AX90L4Y3 for the static and dynamic modes in comparison with data of the catalog and the measured data are given in the table for example. In them $\mathrm{R} 1 *, \mathrm{R} 2 *$ - estimates for the dynamic mode.

Table 1. The parameters.

\begin{tabular}{|c||c|c|c|c|c|c|c|c|}
\hline \multirow{2}{*}{ Data } & \multicolumn{7}{|c|}{ Parameters } \\
\cline { 2 - 9 } & $R_{1}$ & $R_{1}{ }^{*}$ & $L_{1 \sigma}$ & $L_{1}$ & $L_{m}$ & $L_{2}$ & $R_{2}$ & $R_{2}{ }^{*}$ \\
\hline Catalog & \multicolumn{2}{|c|}{3.11} & 0.010 & 0.303 & 0.293 & 0.311 & \multicolumn{2}{|c|}{2.63} \\
\hline Estimates & 3.10 & 3.08 & 0.012 & 0.189 & 0.175 & 0.181 & 2.71 & 2.75 \\
\hline Measurement & \multicolumn{2}{|c|}{3.10} & - & 0.202 & - & - & \multicolumn{2}{|c|}{-} \\
\hline
\end{tabular}

In fig. 1 for the same motor the estimation processes of a rotor active resistance size are given in a static duty, and in fig. 2 - rotor rotation frequency estimation in the dynamic mode is given. The analysis of the received results allowed to do the following conclusions. The experimental check of the developed algorithms confirmed their operability and sufficient accuracy for practical purposes of the received results. At the same time the error of measured parameters did not exceed $10 \%$. 


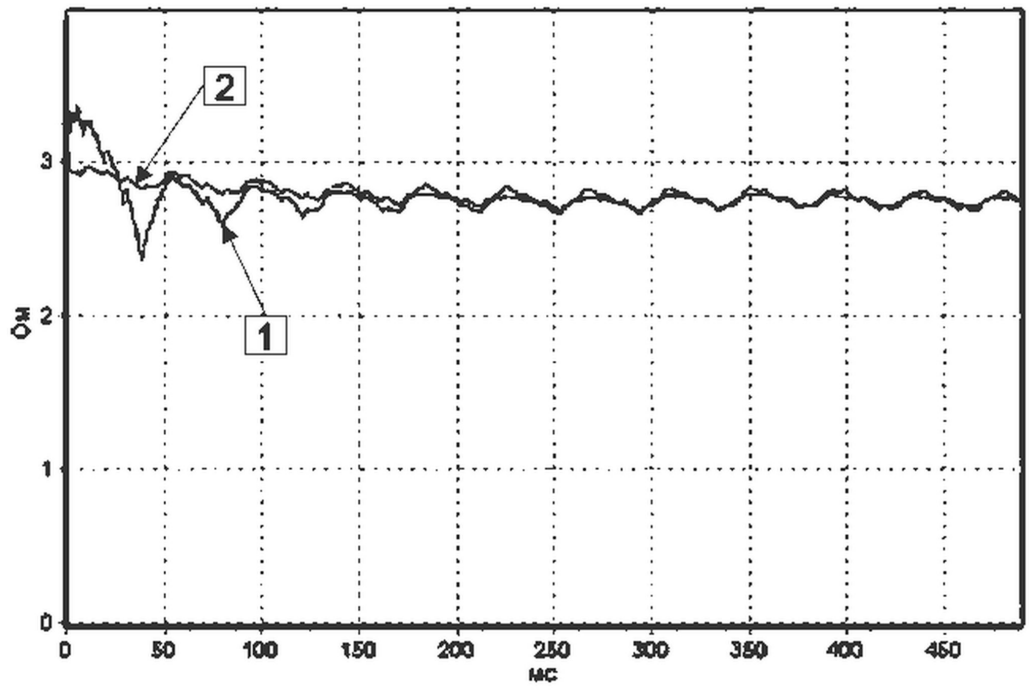

Fig. 1. Estimation process active rotor resistance: 1 - by the first option, 2 - by the second option.

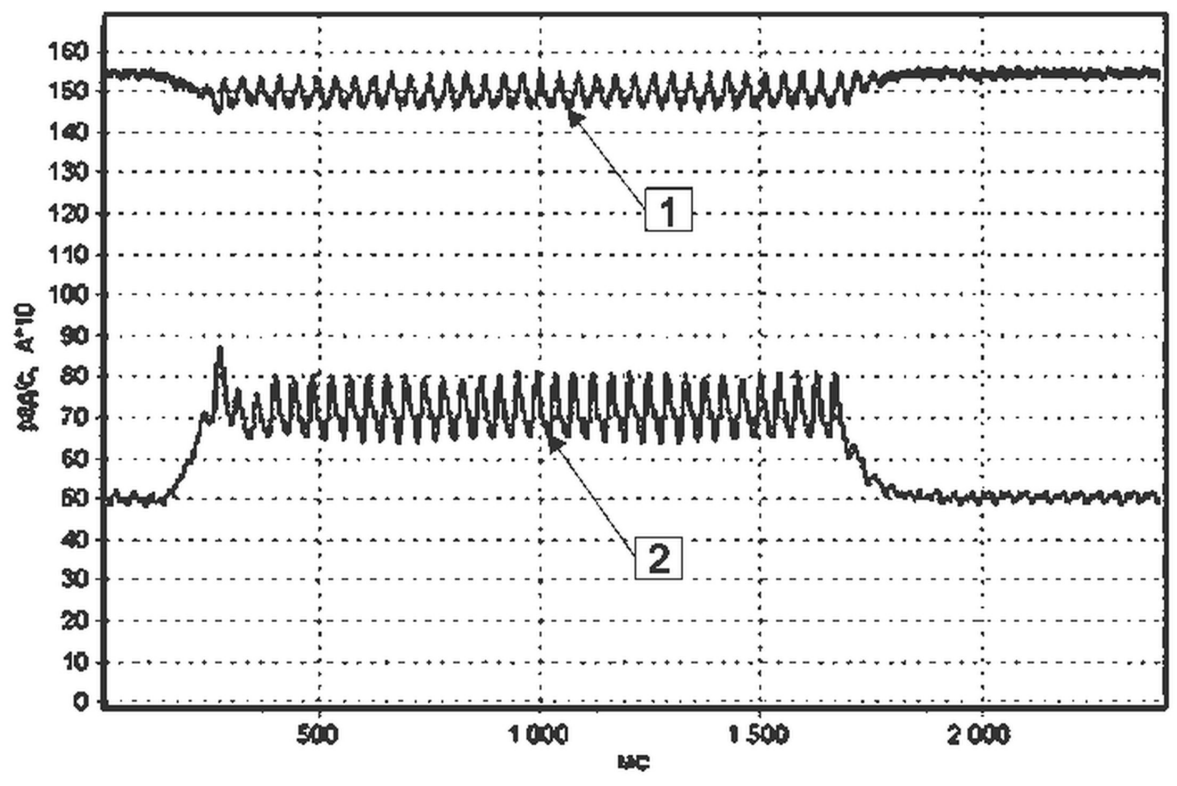

Fig. 2. Process of rotor rotation frequency estimation (1) and the measured stator current (2).

When using of the developed computer system the duration of estimation processes of stator leakage inductance, the rotor magnetic linkage and the magnetization chain is in limits of one period of mains voltage $(20 \mathrm{~ms})$, and rotor inductance sizes and magnetization chain - within 40-50 ms. Estimation of rotor active resistance size on the basis of EKF on the second variant solution also happens within $20 \mathrm{~ms}$, and this variant is accepted by the basic.

The result of the undertaken studies determined that statistical noise properties of the measuring system used for dynamic identification of electric motors quite meet requirements of the used methods. At the same time computing processes have necessary stability. Only use of the sensors measuring phase currents and stator tension is required for 
practical application of the developed identification algorithms allowing to estimate in real time the current values of parameters and variables of an IM state. The motors working in the static modes require also measurement of angular frequency of rotor rotation.

\section{Conclusions}

The received results of theoretical and pilot studies allow to recommend the computer system on the basis of the developed algorithms for monitoring of electromagnetic parameters (active resistance and inductance of the stator and the rotor) and the variables (magnetic linkage of the stator and the rotor, the electromagnetic moment and frequency of rotor rotation) characterizing a condition of asynchronous motors in real time.

The developed algorithms of dynamic IM identification were used for creation of system power - and resource efficient control of electric mining machinery drives constructed on the basis of gradient control of the alternating-current electric drive with optimization of electric power losses and losses capacities in steel and the original speed regulators of end-effector movement providing minimization of variable moments component of resilient forces in transmissions.

This control system was built in a test piece of the electric pull-up drive of a main belt which passed the industrial approbation in underground conditions in the mine named after S.M. Kirov in Kuzbass. At the same time the decrease in a variable component of the resilient moments forces in mechanical subsystems of the electric drive more than for $50 \%$, electric losses - for $7 \%$, and magnetic - for $26 \%$ became available. The technique of the energy saving electric drive creation was accepted for introduction in coal-mining holding company JSC SUEK Kuzbass.

\section{References}

1. F. Alonge, F. D'Ippolo, S. BarberaLa, F.M. Raimondi, Procedings of the IEEE Interniteanal Conference on Control Applications Trieste, 491, 01012 (1998)

2. C. Moons, De.B. Moor, Automatica, 31:8, 1137 (1995)

3. T. Orlowska-Kowalska, IEE Proc. D., 136:6, 324 (1989)

4. L.C. Zai, T.A. Lipo, Conf. Rec. IEEE Ind. Appl. Soc., 177, 100 (1987)

5. J. Liu, I. Kung, H. Chao, Proc. Natl. Sci. Counc. ROC(A), 25:2, 107 (2001)

6. Y. Chen, Electronic Engineering Times, 78, 14 (2001)

7. D.G. Luenberger, IEEE Trans. Mil. Electron, 8, 74 (1964)

8. R.E. Kalman, R. Bucy, Trans. ASME, 83, 95 (1961)

9. Radojka K., Sanja A., Danilo S. FACTA Universitatis, Ser.: Elec. Energ., 18:3, 467 (2005)

10. Wei Tong, Rui Guo, Editorial Office of Optics and Precision Engineeri, 20:10, 2308 (2012)

11. R.Y. Tkachuk, A.S. Glazyrin, V.I. Polishchuk, IFOST Proc., 2, 586 (2012)

12. E.V. Bolovin, A.S. Glazyrin, V.N. Brendakov, SIBCON Proc.,1, 20 (2015)

13. S. Udomsuk, K-L. Areerak, K-N. Areerak, A. Srikaew. Sch. of Electr. Eng., Suranaree Univ. of Techonology (SUT), 48 (2010)

14. A.V. Nesterovskiy, V.G. Kashirskikh, V.M. Zavyalov, I.Y. Semykina, ARPN Journal of Engineering and Applied Sciences, 11:7, 4387 (2016) 
15. V. Kashirskikh, A. Gargayev, V. Zavyalov, I. Semykina, Coal in the 21st Century: Mining, Processing, Safety Proc., 274, 98 (2016)

16. Jigajinni S. Vijaylakshmi, International Journal of Science and Research, 3:8, 1846 (2014)

17. M. S. Z. Salah. Parameters identification of a permanent magnet DC motor (The Islamic University, Gaza, 2009) 